\title{
The effect of physical activity on appetite and food intake in older adults
}

\author{
D. Dericioglu ${ }^{1}$, K. Corbett ${ }^{1}$, L. Methven ${ }^{2}$ and M.E. Clegg ${ }^{1}$ \\ ${ }^{1}$ Hugh Sinclair Unit of Human Nutrition, Department of Food and Nutritional Sciences, University of Reading, \\ Reading, UK and \\ ${ }^{2}$ Food Research Group, Department of Food and Nutritional Sciences, University of Reading, Reading, UK
}

With ageing, older people's appetite and desire to eat decreases, causing body weight and muscle mass to decline which can affect their physical capabilities. Preserving muscle mass and function is essential for maintaining functional independence in older people ${ }^{(1)}$. In addition to the positive effects of physical activity on health and physical function, physical activity is also recommended to enhance appetite in older people that generally have smaller appetites ${ }^{(2,3)}$. However, there is insufficient research to support this advice ${ }^{(4)}$.

The objective of this preliminary study was to investigate the relationship between physical activity levels and body composition, appetite, and food intake in a sample of 25 healthy older men (68.8 (SD 2.9) years; body mass index (BMI) 25 (SD 2.3) $\mathrm{kg} / \mathrm{m} 2$ ) and 25 healthy older women $(69.4$ (SD 2.9) years; BMI 24 (SD 3.1) kg/m2). Participants were divided into two groups based on activity level (high and low) measured using a triaxial accelerometer worn on the wrist for 7 consecutive days. Measurements of height, waist and hip circumference, and body weight, body fat and muscle mass percentage and visceral fat mass were taken using a tape measure and a bioelectrical impedance monitor. Appetite, eating behaviour and nutritional knowledge were examined with self-reported questionnaires: Council on Nutrition, Dutch Eating Behaviour, Three-Factor Eating and General Nutritional Knowledge Questionnaires. Energy intake was assessed by 3-day weighted food diary and appetite was also assessed (using a 100 $\mathrm{mm}$ visual analogue scale) hourly during waking hours for one day.

Independent sample t-tests were performed to compare the participants' characteristics, energy intake, appetite, eating behaviour and nutritional knowledge between the low and high active group. There was no difference in height, weight, BMI, waist circumference, body fat and muscle mass percentage and resting metabolic rate. The hip circumference in the lower activity level group was significantly higher than in the higher activity group $(\mathrm{p}<0.05)$. There was no relationship between physical activity level and appetite, eating behaviour or nutritional knowledge. Energy, protein and fat intake were not significantly different between the groups. Carbohydrate and fibre intake (g) were significantly higher in the higher activity group (232.6 (SD 55); 28.8 (SD 7.5) respectively) compared to the lower activity level group (201.5 (SD 42.3); 22.4 (SD 7.6) respectively) $(\mathrm{p}<0.05)$ ). There were no significant differences between hunger, fullness, wanting and desire to eat food between the groups.

Contrary to the current recommendation, no difference was found between physical activity level and appetite and energy intake in older people. However, a small sample size was used in this preliminary analysis and further data is required to increase confidence in this conclusion.

\section{References}

1. Scientific Advisory Committee on Nutrition (SACN) (2021) SACN statement on nutrition and older adults living in the community.

2. Age UK (2017) Healthy eating overview-Maintaining a healthy weight.

3. NHS (2018) Keeping your weight up in later life.

4. Clegg ME \& Godfrey A (2018) Appetite 128, 145-151. 